\title{
Autonomic Dysfunction Manifesting as Severe Hypertension Following Cobra Envenomation: An Unusual Occurrence
}

\author{
Abhijeet Singh', Viswesvaran Balasubramanian², Nitesh Gupta² \\ 'Department of Pulmonary, Critical care and Sleep Medicine, Vardhaman Mahavir Medical College and Safdarjung Hospital, New Delhi, India \\ 2Department of Respiratory Medicine, Vallabhbhai Patel Chest Institute, University of Delhi, Delhi, India
}

Cite this article as: Singh A, Balasubramanian V, Gupta N. Autonomic Dysfunction Manifesting as Severe Hypertension Following Cobra Envenomation: An Unusual Occurrence. J Emerg Med Case Rep 2018; 9: 51-4.

\begin{abstract}
Introduction: Envenomation due to an elapid snake (krait or cobra) bite is a common life-threatening medical emergency in India and is associated with significant morbidity and mortality. Common cobra (Naja naja) and common krait (Bungarus caeruleus) are the common snake species whose bites are associated with neuroparalysis, which is a key feature of envenomation. Autonomic dysfunction (AD) is another feature of envenomation with varied clinical presentations such as abdominal pain, vomiting, sweating, mydriasis, mild to moderate hypertension (HTN) or hypotension, and cardiac arrhythmia. The occurrence of AD manifesting as severe HTN and tachycardia is unusual following an elapid snake bite. This presentation has been frequently reported with krait envenomation but can also be observed with cobra envenomation.

Case Report: We present the case of a 31-year-old male with common cobra (Naja naja) envenomation presenting with neuroparalysis along with severe HTN and tachycardia. The patient had a gradual and sustained recovery with anti-snake venom and other supportive care.

Conclusion: Increasing incidences of cobra envenomation warrant that understanding its atypical presentation and its early recognition are of paramount importance as they may alter treatment decisions influencing the overall morbidity and mortality.

Keywords: Autonomic dysfunction, cobra, elapid, envenomation, hypertension

Received: 30.09.2017 Accepted: 12.01.2018
\end{abstract}

\section{Introduction}

Neuroparalysis due to envenomation by common cobra (Naja naja) and common krait (Bungarus caeruleus) is a common lifethreatening medical emergency in India (1). Autonomic dysfunction (AD) manifesting as hypertensive (HTN) episodes following envenomation with snakes with neuroparalytic toxins has been reported with krait, but rarely reported with cobra. Timely recognition and appropriate management of AD is of utmost importance to reduce morbidity and mortality. The provision of early anti-snake venom (ASV), selection of appropriate anti-hypertensives, optimization of ventilator support, and other supportive care are warranted. Here we report a rare case of AD manifesting as severe HTN episodes following cobra envenomation with a brief review of literature.

\section{Case Report}

A 31-year-old male who was a farmer presented to our emergency department in the early morning with a history of snake bite $5 \mathrm{~h}$ prior while sleeping on floor; he complained of dizziness, blurring of vision, abdominal pain, vomiting, and breathless- 
ness. His past medical or family history was not contributory as he had never been diagnosed with HTN, diabetes, or kidney diseases. He was a non-smoker and non-alcoholic and was not under any medication, including illicit drugs. He also denied having psychiatric medical complaints. Physical examination revealed a regular heart rate of 120 beats/min, shallow respiratory rate of 28 breaths/min, and blood pressure (BP) of 230/120 mm Hg in the supine position with a strong peripheral pulse and no radioradial or radiofemoral delay. The Glasgow coma scale (GCS) score was 11/15 (E3V4M4), and the saturation was $94 \%$ on room air. Fang marks typical of snakebite were noted at the base of the right great toe. Moreover, 60 min after admission, he developed ptosis, external ophthalmoplegia, and poor gag reflex with difficulty in speech as well as swallowing, are- flexia, and diminished strength in all four limbs as evident on neurological examination. Arterial blood gas analysis revealed hypercapnic respiratory failure with normal alveolar-arterial gradient on room air (pH: 7.33, $\mathrm{PO}_{2}: 65 \mathrm{mmHg}, \mathrm{pCO}_{2}: 50 \mathrm{mmHg}, \mathrm{HCO}_{3}: 26 \mathrm{mEq} / \mathrm{L}$, $\mathrm{PAO}_{2}-\mathrm{PaO}_{2}: 10 \mathrm{mmHg}$ ). He became cyanotic, and his GCS score started declining. He was intubated in emergency under sedation with $4 \mathrm{mg}$ intravenous (IV) midazolam as well as $50 \mu \mathrm{g}$ IV fentanyl and then immediately shifted to the respiratory intensive care unit (ICU) for mechanical ventilation. An examination of the cardiovascular system revealed persistent tachycardia. Respiratory and abdominal examinations were unremarkable. Fundus examination revealed no abnormalities. Laboratory tests including complete blood count, serum electrolytes, hepatic and renal function tests, coagulation pro-

Table 1. Characteristics of cases reporting incidence and outcome of AD manifesting as HTN following a snake bite

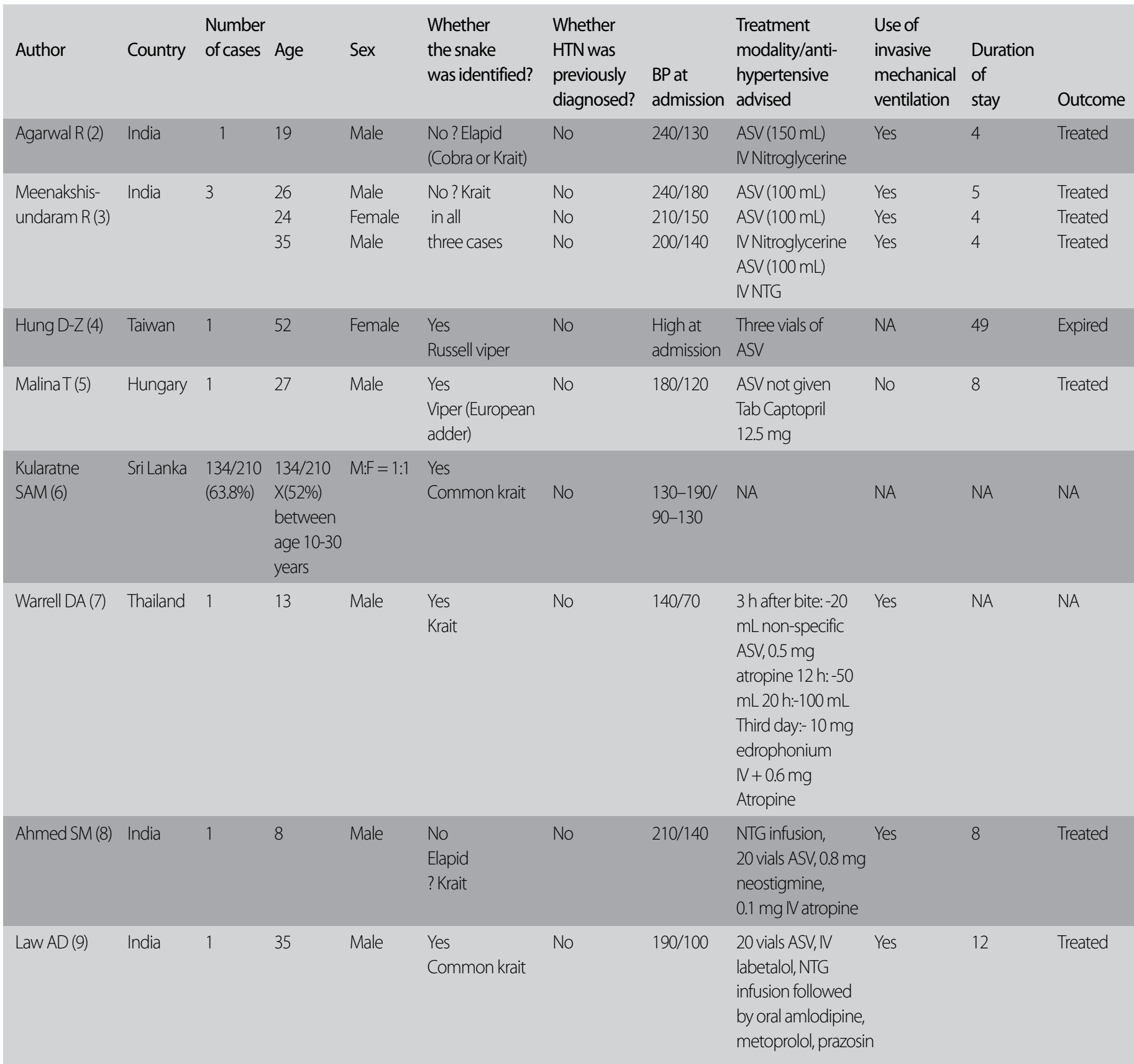


file, and toxicology screen were unremarkable. A clinical diagnosis of neurotoxic snake envenomation was made. The snake was identified as a cobra by the patient's relatives at the site where the patient was bitten. Ten vials (100 mL) of polyvalent ASV (Bengal Chemicals, Calcutta, India) were immediately administered after the establishment of the diagnosis without any complication or evidence of a reaction. The same dose (10 vials) was repeated after 30 minutes with a cumulative dose of 20 vials ( $200 \mathrm{~mL}$ ) as there was no response response to the initial dose of 10 vials $(100 \mathrm{~mL}$ ) of polyvalent ASV. Neostigmine $0.5 \mathrm{mg}$ at 30-min intervals preceded by atropine was also instilled six times. Persistent tachycardia (heart rate: 110-130 beats/ min) as well as high BP (200-240 mmHg systolic and 120-140 mmHg diastolic) with adequate sedation and even paralysis after admission to respiratory ICU was observed. Drug doses used for this patient weighing $60 \mathrm{~kg}$ included a continuous infusion of IV fentanyl 2-5 $\mathrm{\mu g} /$ $\mathrm{kg} / \mathrm{h}$ and vecuronium $0.1 \mathrm{mg} / \mathrm{kg}$ bolus $6 \mathrm{mg}$ followed by $1 \mu \mathrm{g} / \mathrm{kg} /$ min. The etiology of the severe HTN was not immediately clear, and further work up was performed to rule out secondary causes. An electrocardiogram revealed sinus tachycardia without any evidence of left ventricular hypertrophy. Two-dimensional echocardiography revealed normal heart chambers and left ventricular ejection fraction. Urinalysis showed no proteinuria. Chest radiograph; abdominal ultrasound, including renal Doppler ultrasound; and head as well as abdominal computed tomography reports were found to be noncontributory toward HTN. Urinary catecholamine and serum renin/ aldosterone levels were unremarkable. Nitroglycerin (NTG) IV infusion drip with continuous intra-arterial monitoring was initiated to control HTN. During the next $12 \mathrm{~h}$, the patient's neurological status and HTN gradually improved; thus, the NTG infusion was tapered and terminated after $24 \mathrm{~h}$. He was extubated after 3 days of mechanical ventilation and subsequently discharged after 6 days of hospital stay; his BP was recorded to be within normal limits at discharge. He was found to be perfectly healthy and normotensive during the follow-up. Written informed consent was obtained from the patient for presenting this report.

\section{Discussion}

Snake bite is a common life-threatening medical emergency in India and is associated with significant morbidity and mortality. It is estimated that 35,000 people die of snake bite every year in India, with most of the fatalities attributed to delayed access to medical care (1). The common cobra (Naja naja), Russell's viper (Dabiola russelii), saw-scaled viper (Echis carinatus), and common krait (Bungarus caeruleus) are the four highly venomous snakes of the approximately 13 known species of snakes in India. The neurotoxicity induced by elapid snake bites may be predominantly attributed to the neuromuscular blockade due to presynaptic toxins present in krait venom and postsynaptic toxins present in cobra venom, with most elapids containing both pre- and postsynaptic toxins. Envenomation with these elapid snakes manifests as ptosis, external ophthalmoplegia, pharyngeal muscle paralysis, and respiratory and generalized muscle paralyses requiring mechanical ventilation and is associated with an overall mortality of 3.5\% (2). AD manifesting as unexplained tachycardia or bradycardia, HTN or hypotension, pupillary abnormalities, diaphoresis, lacrimation, salivation, vomiting, abdominal pain, paralytic ileus, constipation, and markedly exaggerated circulatory responses to innocuous stimuli such as oral suction and posture change is rare; however, it has been re- ported in literature in patients with snake bite. A brief review of the clinical profile of and outcomes in patients with snake bite leading to neuroparalysis as well as AD leading to HTN is presented in Table 1 (2-9). Most of the cases of AD due to snake bite are attributed to krait bites, with a negligible number of cases attributed to viper. In the present case, the patient was bitten by an elapid snake identified as cobra; he developed neuroparalysis, requiring mechanical ventilation, along with severe HTN as well as tachycardia but had a favorable outcome due to timely initiation of ASV and other supportive measures. In krait bite cases, inhibition of neurally mediated release of norepinephrine by bungarotoxins results in sympathetic overactivity with or without decreased parasympathetic activity, leading to AD manifesting as HTN, whereas in viper bite cases, AD manifesting as HTN is attributed to cardiotoxins, which have the potential to act at or via autonomic synapses (4-9). However, AD due to cobra bites is rarely reported. In the present case, the snake was identified as cobra and the presence of fang marks was confirmed. Various factors support that AD was responsible for occurrence of persistent tachycardia and HTN in our patient such as absence of anxiety at the time of bite which occurred during sleep, persistence despite adequate pain relief, control of hypoxia and sedation during mechanical ventilation and also ruling out of other secondary causes of HTN. This unusual manifestation of cobra bite may be attributed to marked variability in venom composition between and within snake species, contributing to varied toxicity between species and varied clinical manifestations following envenomation. In studies involving animal subjects, other pathogenic mechanisms responsible for AD have been identified but with limited evidence $(6,7,9,10)$. Further research is required in humans in order to describe various pathogenic mechanisms responsible for $A D$ manifesting as HTN due to elapid snake bite particularly cobra. The most important manifestation of the cardiac effects of cobra venom arise from alterations in electrical or contractile properties of the heart. The effect of Egyptian cobra (Naja haje) on BP and chronotropic response of the heart might be dose dependent as reported in a study based on an experiment conducted on rats (10). In that study, a hypothesis was inferred that the differences in the concentration of the neurotoxic and cardiotoxic components of the venom in the blood of envenomated rats affect the entire vascular system, regulating the mean arterial $\mathrm{BP}$ and cardiac muscles in different ways. Elevated BP recorded after the injection of the high-dose venom increases the firing rate of the baroreceptor nerves and results in a decreased output from the sympathetic regions and an increased output from the parasympathetic regions of the brain. A decrease in the sympathetic discharge to the periphery decreases the heart rate. Treatment in such situations involves appropriate ventilator strategies and adequate supportive care. Management of HTN is of utmost importance as complications occurring from severe or uncontrolled HTN may alter the overall course and outcome of the illness. Although the pharmacological management of HTN in these situations is controversial, ASV remains the treatment of choice along with other supportive measures. Beta-blockers should be avoided as they precipitate severe alpha-agonist activity by blocking beta-receptors (3). However, a combination of alpha- and beta-blockers may be considered. Non-adrenergic blocking agents, such as IV NTG, can be used because their dosing can be titrated to the desired effect. In the present case, HTN was managed with IV NTG. At the 
time of discharge, the patient was normotensive, suggesting cobra bite venom was responsible for hypertensive episodes in this patient. Based on demographic and clinical profiles of snake bite envenomation from countries such as Turkey, it is evident that increased awareness regarding such complications among public as well as health care workers could reduce mortality (11). The provision of sufficient as well as good-quality ASV and training of health care workers in managing such cases are considered to be essential steps in regions where snake bite envenomations are common.

\section{Conclusion}

Autonomic dysfunction manifesting as HTN and tachycardia, although reported in krait bite cases, is reported less frequently in cobra bite cases. Understanding this atypical manifestation and its early recognition is of paramount importance for physicians as this manifestation may alter treatment decisions influencing the overall morbidity and mortality.

Informed Consent: Written informed consent was obtained from the parents of the patient who participated in this study.

Peer-review: Externally peer-reviewed.

Author Contributions: Concept - A.S.; Design - A.S., N.G.; Supervision - A.S., N.G.; Resources - A.S., V.B.; Materials - A.S., V.B.; Data Collection and/or Processing - A.S., V.B.; Analysis and/or Interpretation - A.S., N.G.; Literature Search - A.S., N.G.; Writing Manuscript - A.S., V.B.; Critical Review - A.S., N.G.; Other - A.S., V.B.

Conflict of Interest: The authors have no conflict of interest to declare.

Financial Disclosure: The authors declared that this study has received no financial support.

\section{References}

1. Kasturiratne A, Wickramsinghe AR, DeSilva N, Gunawardena NK, Pathmeswaran A, Premaratna R, et al. The global burden of snakebite: A literature analysis and modelling based on regional estimates of envenoming and deaths. PLoS Med 2008; 5: e218. [CrossRef]

2. Agarwal R, Aggarwal AN, Gupta D. Elapid snakebite as a cause of severe hypertension. J Emerg Med. 2006; 30: 319-20. [CrossRef]

3. Meenakshisundaram R, Senthilkumaran S, Grootveld M, Thirumalaikolundusubramanian P. Severe hypertension in elapid envenomation. J Cardiovasc Dis Res 2013; 4: 65-7. [CrossRef]

4. Hung DZ, Wu ML, Deng JF, Yang DY, Lin-Shiau SY. Multiple thrombotic occlusions of vessels after Russell's viper envenoming. Pharmacol Toxicol 2002; 91: 106-10. [CrossRef]

5. Malina T, Krecsak L, Warrell DA. Neurotoxicity and hypertension following European adder (Vipera berus berus) bites in Hungary: case report and review. QJM 2008; 101: 801-6. [CrossRef]

6. Kularatne SA. Common krait (Bungarus caeruleus) bite in Anuradhapura, Sri Lanka: a prospective clinical study, 1996-98. Postgrad Med J 2002; 78: 276-80. [CrossRef]

7. Warrell DA, Looareesuwan S, White NJ, Theakston RDG, Warrell MJ, Kosakarn W, et al. Severe neurotoxic envenoming by the Malayan krait Bungarus candidus (Linnaeus): response to anti-venom and anti-cholinesterase. Br Med J (Clin Res Ed). 1983; 286: 678-80. [CrossRef]

8. Ahmed SM, Khan M, Zaka-ur-Rab Z, Nadeem A, Agarwal S. Hypertensive encephalopathy following snake bite in a child: A diagnostic dilemma. Indian J Crit Care Med 2013; 17: 111-12. [CrossRef]

9. Law AD, Agrawal AK, Bhalla A. Indian common krait envenomation presenting as coma and hypertension: A case report and literature review. J Emerg Trauma Shock 2014; 7:126-8. [CrossRef]

10. Omran MA, Abdel-Nabi IM. Changes in the arterial blood pressure, heart rate and normal ECG parameters of rat after envenomation with Egyptian cobra (Naja haje) venom. Hum Exp Toxicol 1997; 16: 327-33. [CrossRef]

11. Cesaretli Y, Ozkan O. Snakebites in Turkey: epidemiological and clinical aspects between the years 1995 and 2004. Rev Inst Med Trop Sao Paulo 2010; 52: 215-20. [CrossRef] 